\title{
TOPS: a small space telescope using phase induced-amplitude apodization (PIAA) to image rocky and giant exo- planets
}

Olivier Guyon, James R. P. Angel, Charles Bowers, James Burge, Adam Burrows, et al.

Olivier Guyon, James R. P. Angel, Charles Bowers, James Burge, Adam Burrows, Johanan Codona, Thomas Greene, Masanori lye, James Kasting, Hubert Martin, Donald W. McCarthy, Victoria Meadows, Michael Meyer, Eugene A. Pluzhnik, Norman Sleep, Motohide Tamura, Domenick Tenerelli, Robert Vanderbei, Bruce Woodgate, Robert A. Woodruff, Neville J. Woolf, "TOPS: a small space telescope using phase induced-amplitude apodization (PIAA) to image rocky and giant exo-planets," Proc. SPIE 6693, Techniques and Instrumentation for Detection of Exoplanets III, 66930J (19 September 2007); doi: 10.1117/12.735160

SPIE Event: Optical Engineering + Applications, 2007, San Diego, California, United States 


\title{
TOPS: A small space telescope using phase induced-amplitude apodization (PIAA) to image rocky and giant exo-planets
}

\author{
Olivier Guyon ${ }^{a, b}$, James R.P. Angel ${ }^{b}$, Charles Bowers ${ }^{c}$, James Burge ${ }^{c}$, Adam Burrows ${ }^{c}$, \\ Johanan Codona ${ }^{c}$, Thomas Greene ${ }^{d}$, Masanori Iye ${ }^{e}$, James Kasting ${ }^{f}$, Hubert Martin ${ }^{b}$, Donald \\ W. McCarthy, Jr. ${ }^{b}$, Victoria Meadows ${ }^{g}$, Michael Meyer ${ }^{b}$, Eugene A. Pluzhnik ${ }^{a}$, Norman Sleep ${ }^{h}$ \\ , Motohide Tamura ${ }^{e}$, Domenick Tenerelli ${ }^{i}$, Robert Vanderbei ${ }^{j}$, Bruce Woodgate ${ }^{c}$, Robert A. \\ Woodruff ${ }^{i}$, Neville J. Woolf ${ }^{b}$ \\ ${ }^{a}$ Subaru Telescope, NAOJ, 650 N. A'ohoku Pl., Hilo, HI, 96720, USA; \\ ${ }^{b}$ Steward Observatory, The University of Arizona, 933 N. Cherry Ave., Tucson, AZ 87521, USA \\ ${ }^{c}$ Goddard Space Flight Center, Greenbelt, MD 20771, USA \\ ${ }^{d}$ Ames Research Center, Moffett Field, CA 94035, USA \\ ${ }^{e}$ National Astronomical Observatory of Japan, Osawa 2-21-1, Mitaka, Tokyo 181-8588, Japan

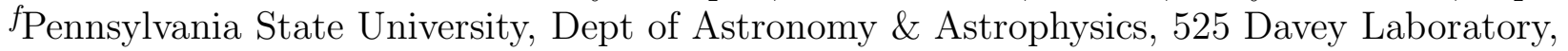 \\ University Park, PA 16802, USA \\ ${ }^{g}$ IPAC, Caltech, 770 S. Wilson, Pasadena, CA 91125, USA \\ ${ }^{h}$ Stanford University, Mitchell Building, room 373A, Stanford, CA 94305-2215, USA \\ ${ }^{i}$ Lockheed Martin Space Corporation, P.O. Box 179, Denver, CO 80201-0179, USA \\ ${ }^{j}$ Princeton Univ., ORFE, Ace-42, E-Quad, Princeton, NJ 08544, USA
}

\begin{abstract}
The Telescope to Observe Planetary Systems (TOPS) is a proposed space mission to image planetary systems of nearby stars simultaneously in a few wide spectral bands covering the visible light $(0.4-0.9 \mu \mathrm{m})$. It achieves its power by combining a high accuracy wavefront control system with a highly efficient Phase-Induced Amplitude Apodization (PIAA) coronagraph which provides strong suppression very close to the star (within $2 \lambda / \mathrm{D}$ ). The PIAA coronagraphic technique opens the possibility of imaging Earthlike planets in visible light with a smaller telescope than previously supposed. If sized at $1.2-\mathrm{m}$, TOPS would image and characterize many Jupiter-sized planets, and discover $2 R_{E}$ rocky planets within habitable zones of the $\approx 10$ most favorable stars. With a larger 2 -m aperture, TOPS would have the sensitivity to reveal Earth-like planets in the habitable zone around $\approx 20$ stars, and to characterize any found with low resolution spectroscopy. Unless the occurrence of Earth-like planets is very low $\left(\eta_{\oplus}<\sim 0.2\right)$, a useful fraction of the TPF-C scientific program would be possible with aperture much smaller than the baselined 8 by $3.5 \mathrm{~m}$ for TPF, with its more conventional coronagraph. An ongoing laboratory experiment has successfully demonstrated high contrast coronagraphic imaging within $2 \lambda$ /d with the PIAA coronagraph / focal plane wavefront sensing scheme envisioned for TOPS.
\end{abstract}

Keywords: Coronagraphy, Adaptive Optics, Space Telescopes, Exoplanets

\section{INTRODUCTION}

There are now about 250 known exoplanets, nearly all of them identified by indirect detection techniques. Within the next decade, our understanding of exoplanetary system architectures will be further enhanced by groundbased imaging of young massive exoplanets and protoplanetary disks with "Extreme-AO" systems, and indirect detection of a large number of planets ranging from Earth mass to gas giants with radial velocity, transit and

Further author information: (Send correspondence to Olivier Guyon.)

Olivier Guyon.: E-mail: guyon@naoj.org, Telephone: 18089345901

Techniques and Instrumentation for Detection of Exoplanets III, edited by Daniel R. Coulter

Proc. of SPIE Vol. 6693, 66930J, (2007) · 0277-786X/07/\$18 · doi: 10.1117/12.735160 
microlensing searches. While detection of Earth-like planets is still out of reach for current instruments, it now appears almost certain that such planets exist, and may even be in habitable zones of nearby stars. ${ }^{1}$ Identification and characterization of our closest Earth-like neighbors through direct visible imaging and spectroscopy will be crucial to understanding the formation and evolution of habitable planets around normal stars, and to look for evidence of life on these planets. This is however very challenging due the $\approx 10^{10}$ contrast in visible light and $\approx 0.1 "$ separation between the star and its Earth-like planet(s).

We present in this paper what we believe is a very promising approach to reach this goal: a highly efficient coronagraphic visible-light space telescope of modest size $(\approx 2 \mathrm{~m})$. The coronagraphic technology adopted would allow operation near the limit of sensitivity imposed by fundamental physics. The science goals such a capability could address include: characterizing the physical properties of a large number of gas giant planets in the solar neighborhood; search for zodiacal dust clouds around nearby stars down to solar system levels; and the frequency and nature of terrestrial planets around the nearest 20 stars. Together, these surveys could enable us, for the first time, to understand the connections between gas giant and terrestrial planets around sun-like stars, enabling us to place our solar system in context.

To overcome the enormous star/planet contrast challenge, TOPS utilizes the most efficient coronagraphic and wavefront control techniques recently developed. The coronagraph adopted is the highly efficient Phase-Induced Amplitude Apodization (PIAA) coronagraph, described in $\S 2$. The wavefront control strategy, described in $\S 3$, relies upon accurate sensing of residual light within and after the coronagraph, and a 2-step wavefront correction (primary mirror and fine DM). Expected performance for both detection and characterization of exoplanets is quantified in $\S 4$.

\section{CORONAGRAPH}

We give in this section a brief description of the PIAA coronagraph concept and performance. More detailed description of the technique can be found in several recent publications. ${ }^{3-10}$

\subsection{PIAA CORONAGRAPH PRINCIPLE}

The goal of a coronagraph is to remove the diffraction features in the on-axis Point Spread Function (PSF) of a telescope. Since these diffraction features are due to the amplitude distribution in the pupil (in a "classical" circular pupil telescope, the bright Airy rings are due to the sharp edges of the pupil), a coronagraphic effect may be obtained by modifying ("apodizing") the intensity distribution in the pupil. This can be done by placing a partially transmissive mask in the pupil plane, which unfortunately needs to remove most of the light at the edges of the pupil.

A much more efficient approach, the PIAA coronagraph ${ }^{3}$ illustrated in Figure 1, uses geometric reflection on aspheric mirrors to perform a lossless apodization of the telescope beam. This lossless apodization preserves the sensitivity and angular resolution of the telescope, and offers an Inner Working Angle (IWA) which is slightly less than $2 \lambda / \mathrm{D}$ at the $10^{10}$ contrast. As detailed in $\S 2.3$, the beam apodization is shared between the aspheric PIAA optics, which performs most of the apodization, and conventional apodizer(s), which only remove(s) a small fraction of the light.

The aspheric apodizing optics introduce very large optical aberrations for off-axis sources, and limit the practical field of view to approximately $10 \lambda / \mathrm{D}$. This can be efficiently solved by adding post-coronagraphic correcting optics, which can be physically very small and do not need to be of "coronagraphic" quality.

\subsection{CORONAGRAPH PERFORMANCE}

A full PIAA coronagraph system, as shown conceptually in Figure 1, offers simultaneously:

1. High contrast at small angular separation. The PIAA achieves high contrast (limited by wavefront control accuracy) at small IWA (from $1.5 \lambda / d$ to $2 \lambda / d$, depending on the exact design adopted). The ability to search for planets at this small angular separation is essential to access the habitable zone of nearby stars with a moderately sized telescope. 


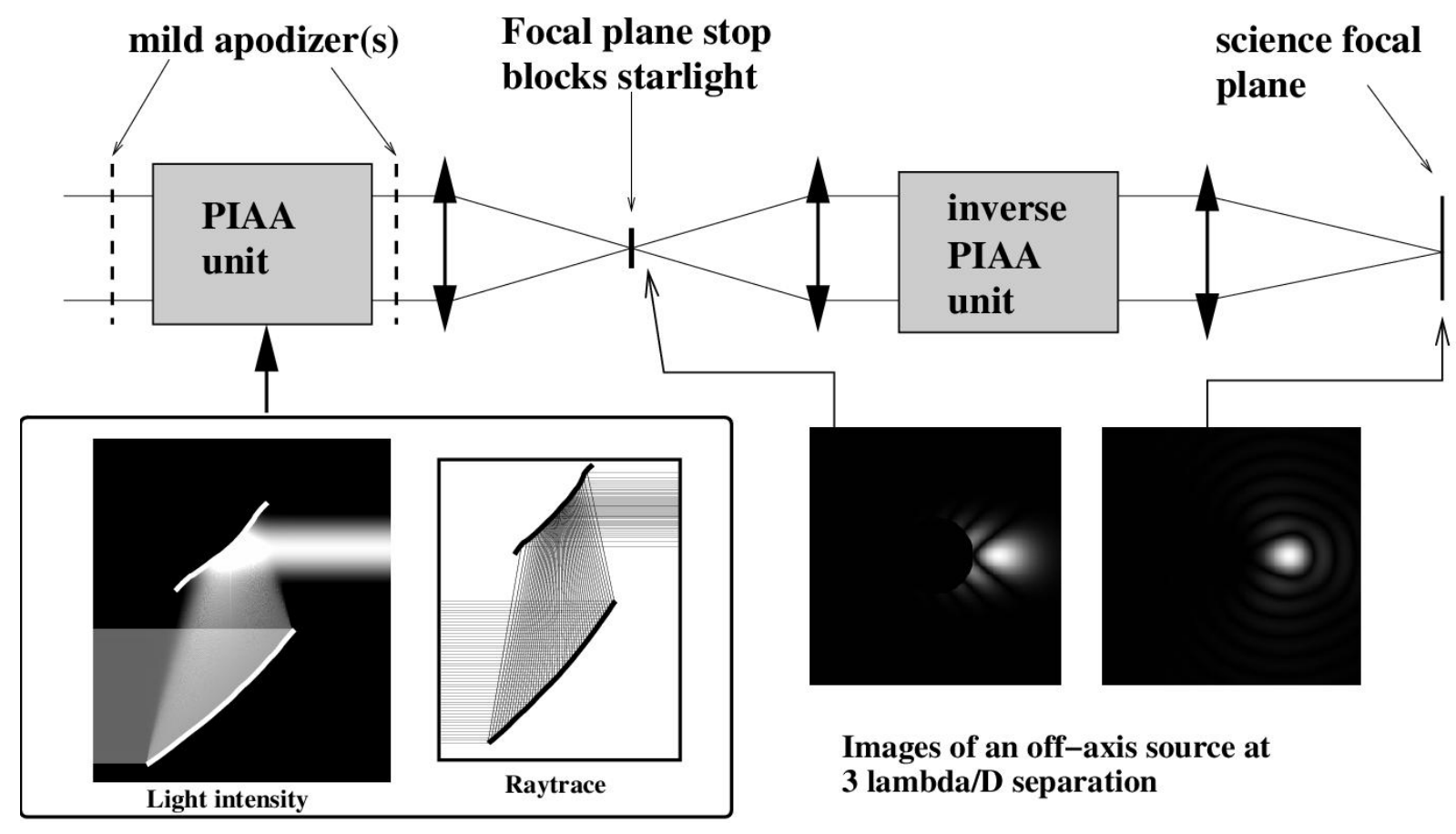

Figure 1. Schematic representation of the PIAAC. The telescope light beam enters from the left and is first apodized by the PIAA unit. Mild apodizer(s) are used to perform a small part of the apodizations, and are essential to both mitigate chromatic diffraction propagation effects and allow for the design of "friendly" aspheric PIAA mirrors. An high contrast image is then formed, allowing starlight to be removed by a small occulter. An inverse PIAA unit is required to "sharpen" the image of off-axis sources.

2. High throughput. The PIAA system offers nearly $100 \%$ throughput and 360 -degree search area. The low intrinsic luminosity of planets imposes strong requirements on the throughput and efficiency of the coronagraph. At and beyond $2 \lambda / d$ (slightly more for a star of large angular diameter), the PIAAC transmits more than $2 / 3$ of the planetary photons, regardless of position angle. This high throughput also enables accurate wavefront control, as the number of photons made available for wavefront sensing is large, and aberrations can therefore be sensed rapidly.

3. Robustness to stellar angular size. Unlike other small-IWA $(<2 \lambda / d)$ coronagraphs, the PIAAC performance is only moderately affected by "small" stellar angular size and small $(\approx 0.01 \lambda / d$ or less $)$ pointing errors, provided that the focal plane occulter is slightly oversized $(\approx 1.8 \lambda / d$ in our baseline design).

4. Preserves the telescope's angular resolution. Thanks to the absence of an undersized Lyot pupil plane stop, the planet light is confined into a single $\lambda / \mathrm{D}$ - wide diffraction core. This is critical to minimize the amount of zodiacal and exozodiacal light mixed with the planet light, and provides additional robustness against confusion with exozodiacal features, coronagraphic leaks, background sources and other planets.

5. Can be designed with high level of achromaticity. Our proposed "hybrid" PIAA design ${ }^{9}$ relies on both PIAA apodization and mild conventional apodization. It can be designed to maintain high contrast simultaneously in a broad spectral band, at the cost of a small loss of light (about 10\%) in the mild apodizer(s).

When all factors are taken into account, the PIAAC offers the same performance level that conventional coronagraphs on a telescope $\approx \mathbf{2}$ times smaller in diameter. ${ }^{11}$ Examples of "conventional" coronagraphs 


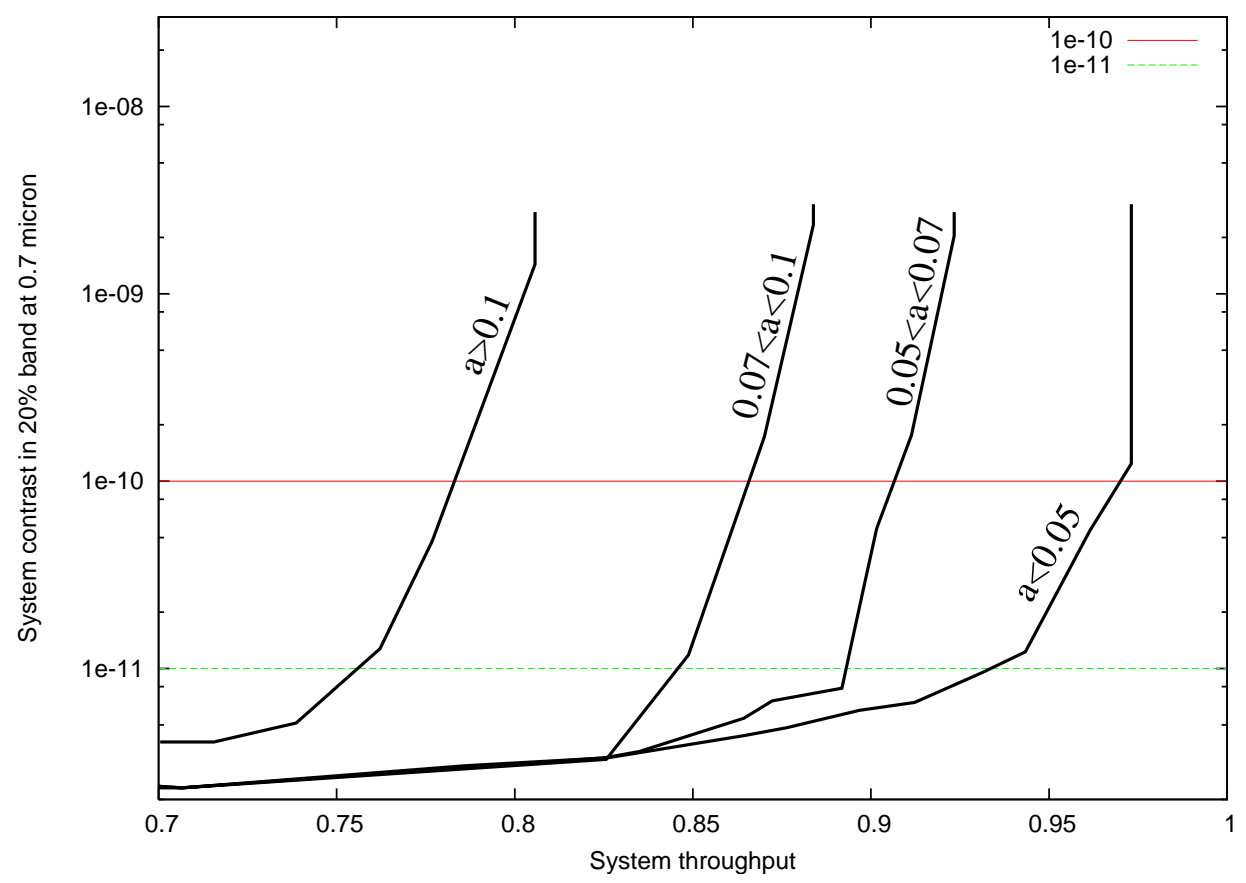

Figure 2. PIAA achromaticity vs. system throughput. The peak contrast (worst PSF contrast encountered outside 2 $\lambda / D)$ for a polychromatic coronagraphic PSF with a $20 \%$ wide band centered at $0.7 \mu \mathrm{m}$ is shown as a function of system throughput. System throughput takes into account losses due to pre-PIAA/post-PIAA apodizers and beam oversizing. Four curves are shown, with different allowed values for the parameter $a$, which is the surface brightness ratio between the center and the edge of the beam delivered by the PIAA optics (without apodizers). The manufacturability of PIAA optics is a function of $a$ : for small values of $a$, PIAA mirror M1 is challenging to manufacture.

include the $8^{\text {th }}$ order band-limited coronagraph ${ }^{12}$ and conventional pupil apodization, ${ }^{2}$ both previously considered for the baseline TPF-C mission.

\subsection{CHROMATICITY \& MANUFACTURABILITY}

Entirely relying upon the PIAA optics to apodize the telescope beam would require very "unfriendly" aspheric mirror shapes, with a strong curvature at the edge of the first PIAA mirror. This would results in both serious manufacturability challenges and strong chromatic diffraction propagation effects ${ }^{8}$ which restrict the spectral bandwidth across which high contrast can be maintained. In a hypothetical pure-PIAA system, the useful spectral bandwidth at the $10^{10}$ contrast would be too small for efficient detection of exoplanets. Since this problem originates from the need to produce the very dark outer edge of the apodized beam, it can be efficiently mitigated by designing PIAA optics with do not fully apodize the beam ${ }^{9}$ (typically leaving the edge of the apodized beam at $1 \%$ of the surface brightness of the center of the apodized beam) and using conventional apodization to complete the apodization. The conventional apodizer may be placed before and/or after the PIAA unit, and can be either a continuous or a binary apodization mask. This "hybrid" PIAA design is adopted for TOPS. The sharing of the apodization between the PIAA aspheric optics and the conventional apodizer is an important parameter in the design of the PIAA coronagraph system, as it defines (1) the chromaticity of the PSF contrast, (2) the manufacturability of the PIAA optics and (3) the system throughput.

We have developed a PIAA diffraction propagation code to quantify how chromaticity, manufacturability and system throughput can be balanced. The code accurately simulates chromatic effects due to diffraction effects created by both the aspheric shape of the PIAA optics and beam-edge effects. A critical design parameter for the PIAA system is the surface brightness $a^{2}$ (normalized to the surface brightness at the center of the beam) of the beam delivered by the aspheric optics. For large values of $a$ : the conventional apodizer needs to remove a 
large fraction of the light at the outer edge of the beam, and the system throughput is therefore low; the optics are more easily manufacturable; the constrast chromaticity should be good. Our design optimization included other free parameters quantifying the profile of the apodized beam delivered by the aspheric optics and the overfilling / edge apodization of PIAA M1 to prevent edge diffraction effects (we note that this also affects the system throughput). Approximately 10000 different designs were tested within reasonable bounds of the design parameters. The beam size within the PIAA system was chosen to be $90 \mathrm{~mm}$, with a $0.9 \mathrm{~m}$ separation between PIAA M1 and PIAA M2 separation. PSF contrast was computed within a $20 \%$ wide band centered on $0.7 \mu \mathrm{m}$.

The results are compiled in Figure 2, which shows, for different constraints on parameter $a$, how PSF chromaticity and system throughput can be chose. The most important result of this study is to show that the PIAA system throughput is likely to be limited by PIAA optics manufacturability rather than by diffraction propagation concerns. With no constraint on manufacturability ( $a<0.05$ curve), a $1 \mathrm{e}-10$ contrast in a $20 \%$ wide band can be achieved with a $97 \%$ system throughput. With a $a>0.05$ constraint on manufactuability, the system throuput drops to $\approx 91 \%$. The practical limit on parameter $a$ imposed by manufacturing constraints is still poorly known. Our first mirror-based PIAA system was made with $a=0.1$, and we are currently working to obtain a second generation PIAA system which may have a smaller $a$ value.

We note that the $20 \%$ wide band requirement used in this section is compatible with the baseline TOPS design, which covers the 0.4 to $0.9 \mu \mathrm{m}$ range in 4 channels, each $20 \%$ wide. Since the chromatic differences between channels are very small in both phase and amplitude, the same PIAA aspheric optics can be used in common to all channels, and chromatic differences between the channels are corrected for by small modifications to the apodizer and mirror shapes (active DM or static mirror) after wavelength separation into 4 channels by dichroics.

\subsection{LABORATORY DEMONSTRATION}

Our current PIAA coronagraph laboratory prototype is located at Subaru Telescope and is co-funded by Subaru Telescope and NASA/JPL. It includes a monochromatic light source (single mode fiber at HeNe), immediately followed by the two PIAA aspheric mirrors (which are designed for a f/15 diverging input beam and deliver a f/15 output converging beam). All optics (PIAA and reimaging optics) are within a temperature-regulated enclosure which provides thermal stability. A 1024 actuators (32x32) MEMs-type actuators, driven by custom-built 16-bit high voltage drivers, is used to correct for residual wavefront aberrations. A phase diversity algorithm is used for wavefront control (the diversity is introduced by the DM itself). In the initial configuration of our experiment, no focal plane mask was blocking starlight, and the apodizer was not located in a pupil plane. Despite these limitations, a 1e-6 contrast was reached at $1.5 \lambda / \mathrm{D}$ from the optical axis (see Figure 3 ).

Ongoing improvements to this laboratory experiment should enable higher contrast: a high accuracy temperature regulation system provides increased stability, and a low-order wavefront sensor is now installed.

A full PIAA system, including MEMs-based wave-front control, is also being developed for the Subaru Telescope. This system will make use of refractive PIAA optics in the near-IR (H-band) and is currently under assembly, with first light expected within 1.5 years. Key TOPS technologies (PIAA coronagraph, low-order wavefront sensing) will be soon tested on this ground-based system, although at a much lower contrast.

\section{WAVEFRONT CONTROL}

To allow $10^{10}$ contrast at $2 \lambda / \mathrm{D}$, the full system must achieve wave-front correction to sub-nm level for the low and mid spatial frequencies. It is therefore critical to design a telescope assembly as stable as possible and to measure low \& mid spatial frequencies during science observations. The combined wavefront control and coronagraphic architecture for TOPS is shown in Figure 4

\subsection{WAVEFRONT CORRECTION}

The wavefront correction for TOPS is done with deformable mirrors (DMs). The number and placement of DMs will be optimized to minimize the total number of DMs and relay optics while maintaining sufficient control to maintain the high coronagraphic contrast in polychromatic light. 

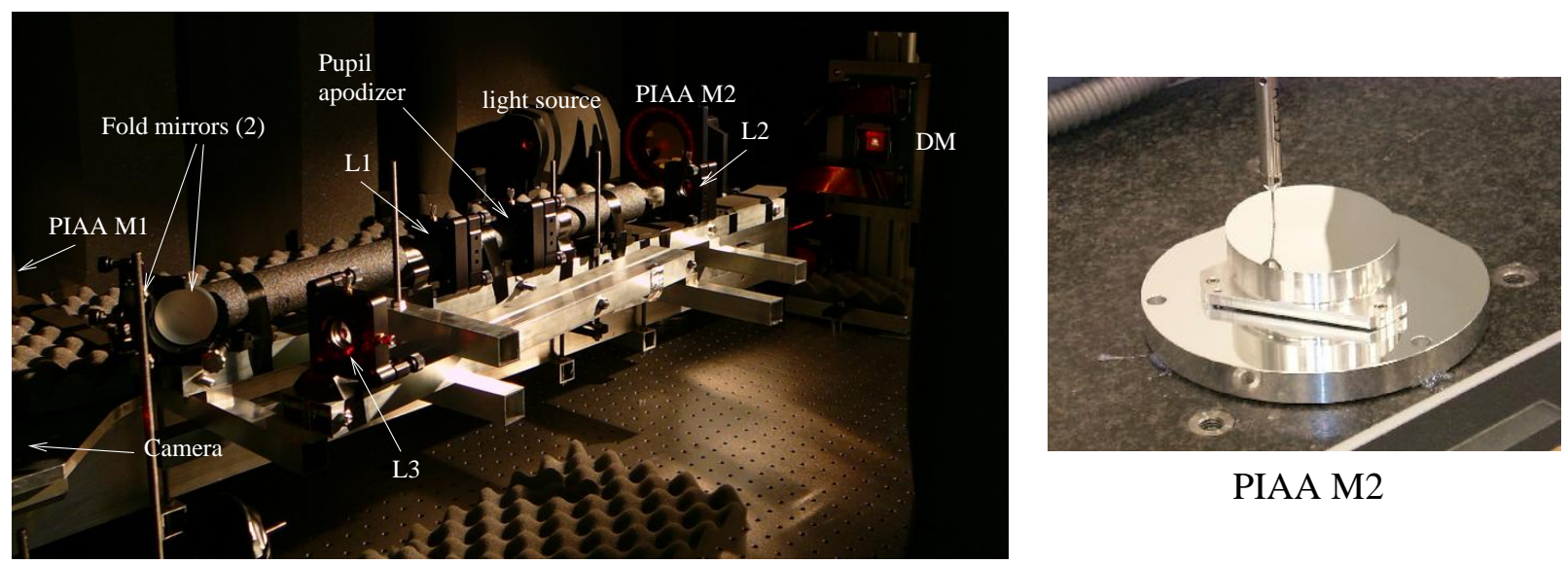

PIAA M2
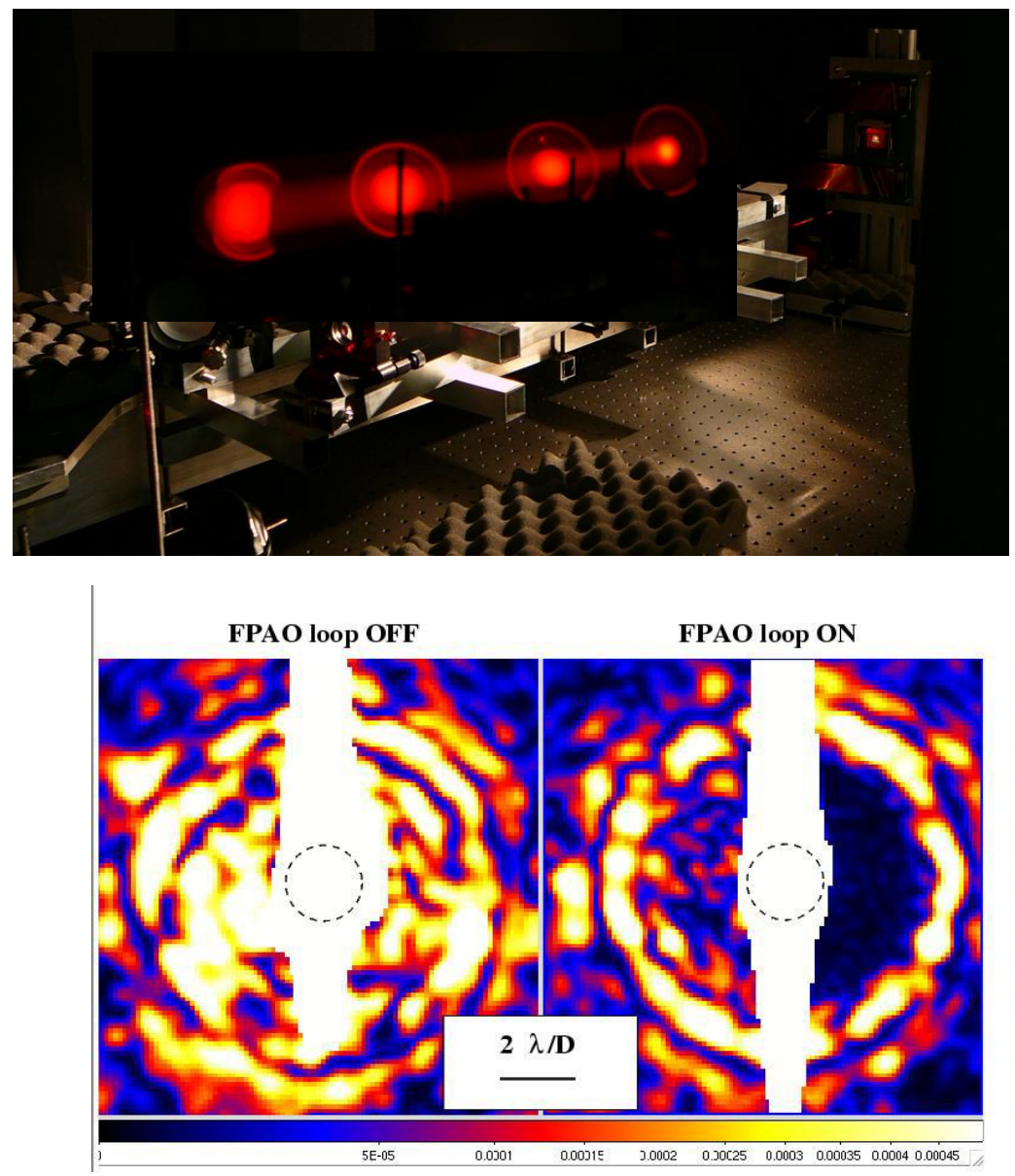

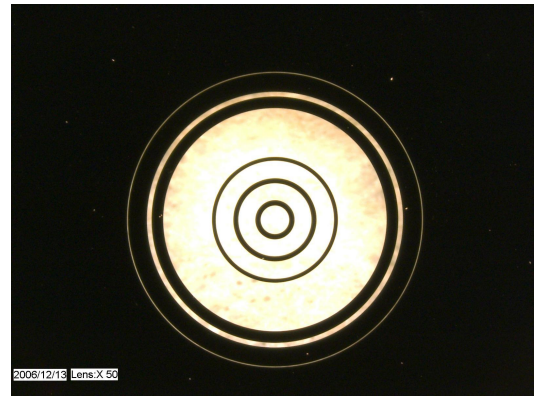

Pupil apodizer

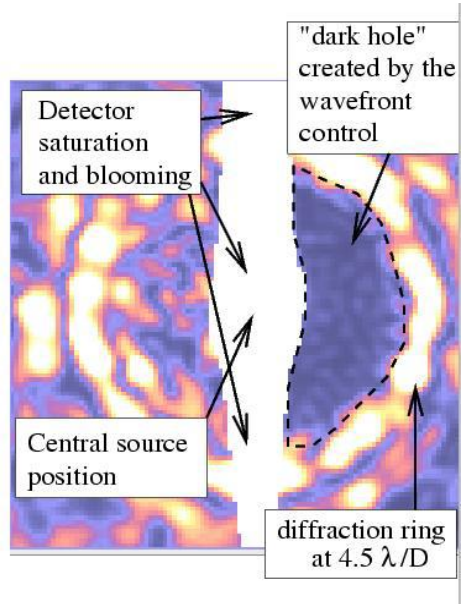

Figure 3. PIAA coronagraph laboratory prototype. The optical layout is shown in the upper left. The beam is apodized (center left) by the PIAA mirrors (upper right) and a conventional apodizer (center right). Thanks to wavefront correction with a deformable mirror, this prototype has reached better than $10^{6}$ contrast at $1.5 \lambda / \mathrm{D}$ separation (bottom). 


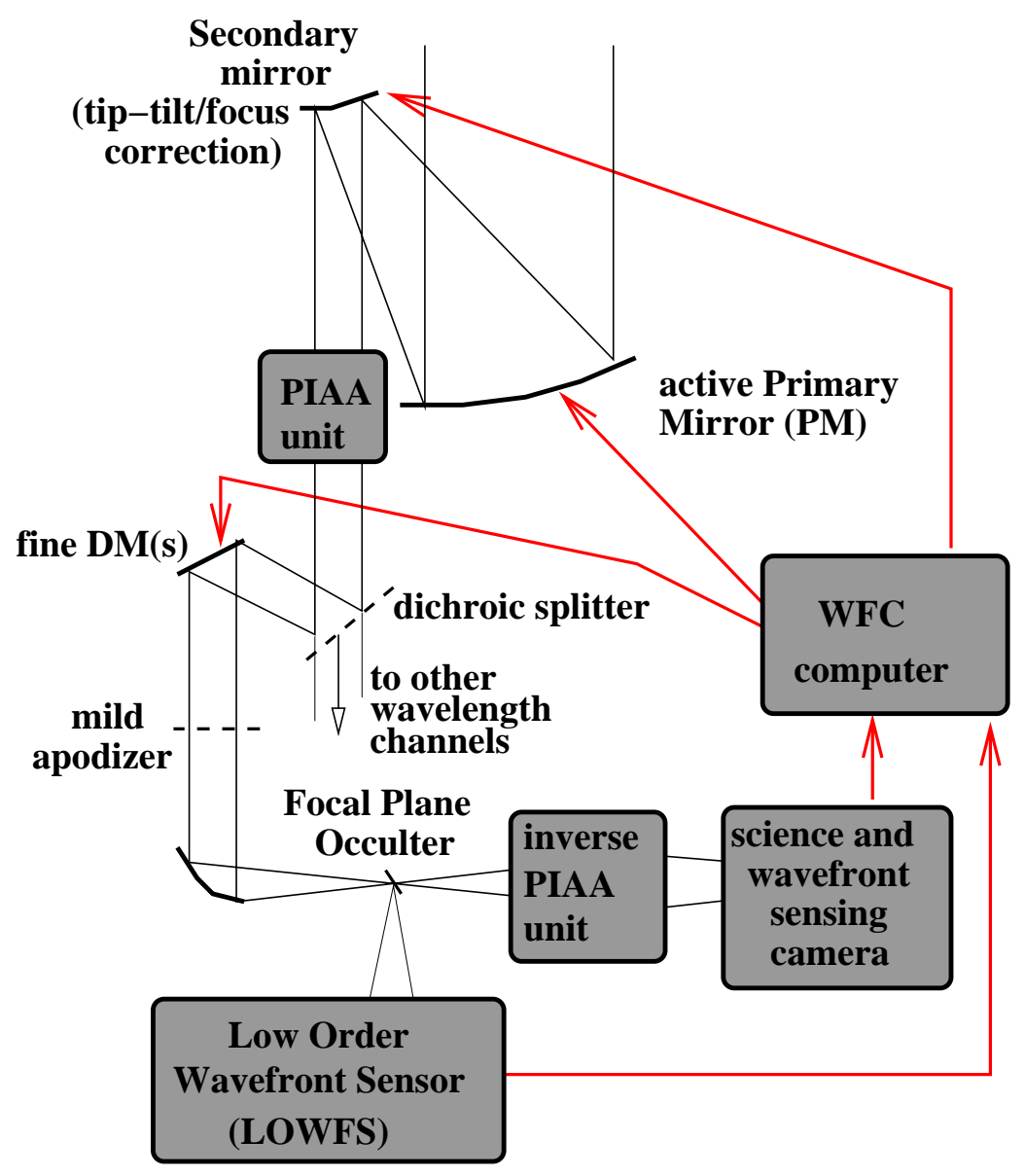

Figure 4. Schematic representation of the combined coronagraphic and wavefront control architecture for TOPS.

The TOPS baseline includes an active primary mirror, which would be thermally actuated. Thermal actuation of an otherwise conventional honeycomb mirror seems to be a very robust approach and is fully compatible with the low stroke/lowspeed requirements of TOPS wavefront control. The mirror will be structured as a lightweight honeycomb sandwich. and figured in just the same way as current space telescope primaries, to a surface accuracy of $5 \mathrm{~nm}$ RMS. Glass of smal but finite expansion coefficient will be used, so that shape changes can be introduced with thermal gradients to correct the redisual errors of low order. Temperatures will be set locally by radiative coupling to a thermal control surfaces in each honeycomb cell. Further details of the active primary concept are given in these proceedings in the paper by Angel, Cuerden, Guyon, Kang \& Stahl.

The TOPS architecture includes several wavelength channels (four in our baseline concept), separated by dichroic(s) immediately after the common path PIAA optics. This enables simultaneous observations in four channels covering a wide spectral range, which is critical to minimize exposure time and quickly acquire valuable spectrophotometry information. This also allows better coronagraphic performance, as the focal plane occulter size can be optimally chosen for each wavelength range. Within each wavelength channel, a small DM performs further correction, and is needed to correct spatial frequencies too high for the active primary mirror. This DM is located after the PIAA unit and can therefore be driven to create a dark hole in the coronagraphic focal plane where the focal plane occulter is placed. To maximize contrast achromaticity, this DM is conjugated near the primary mirror, where most time-variable wavefront aberrations are expected. Most of the wavefront errors from smaller optics can be corrected with "static DMs": precision figuring of a few selected optical surfaces will be done to remove system wavefront errors prior to launch. Remaining static errors should be small enough to 
efficiently be removed by the fine DM within each spectral channel.

\subsection{WAVEFRONT SENSING AND CONTROL}

Wavefront sensing requirements are summarized in table 2. Wavefront sensing is performed at both the science focal plane ${ }^{13}$ and in a dedicated low-order wavefront sensor (described in §3.2.2).

Table 1. Wavefront control requirements, mitigation scheme, sensing and correction strategy for $10^{10}$ contrast on a PIAA-based 2-m diameter telescope. A coronagraph system including PIAA, focal plane occulter and inverse PIAA was simulated at $550 \mathrm{~nm}$ to derive these requirements. The sampling time necessary to measure the corresponding level of aberration at $\mathrm{SNR}=5$ is given here for a $m_{V}=5$ star.

\begin{tabular}{|l|l|l|l|l|}
\hline Aberration & $\begin{array}{l}\text { Required } \\
\text { accuracy } \\
\text { (RMS per } \\
\text { mode) }\end{array}$ & Mitigation & $\begin{array}{l}\text { Sensor \& } \\
\text { SNR = } \\
\text { sampling } \\
\text { time }\end{array}$ & correction \\
\hline \hline Pointing & $0.9 \mathrm{~nm}$ & $\begin{array}{l}\text { Disturbance } \\
\text { Free Payload }\end{array}$ & $\begin{array}{l}\text { LOWFS } \\
0.2 \mathrm{sec}\end{array}$ & $\begin{array}{l}\text { spacecraft } \\
\text { pointing }\end{array}$ \\
\hline Focus & $43 \mathrm{pm}$ & Spacecraft & $\begin{array}{l}\text { LOWFS } \\
\text { \& OTA design }\end{array}$ & $\begin{array}{l}\text { OTA } \\
\text { focus }\end{array}$ \\
\hline Astigmatism & $70 \mathrm{pm}$ & stable OTA & $\begin{array}{l}\text { LOWFS } \\
\text { stable PM }\end{array}$ & PM \\
& & sec & \\
\hline $\begin{array}{l}\text { Mid } \\
\text { spatial } \\
\text { frequencies }\end{array}$ & $1.5 \mathrm{pm}$ & stable OTA & $\begin{array}{l}\text { Science } \\
\text { CCD }(\mathrm{s})\end{array}$ & $\begin{array}{l}\text { PM } \\
+ \text { DM } \\
2 \text { min }\end{array}$ \\
\hline
\end{tabular}

\subsubsection{FOCAL PLANE WAVEFRONT SENSING}

In this scheme, akin to phase diversity, known offsets are sent to the DMs upstream of the coronagraph occulter. The corresponding variations in the post-coronagraphic image speckles provide the signal to reconstruct the precoronagraph wavefront, or, equivalently, the complex amplitude of the focal plane speckle field. The science focal plane array is immune to non-common path errors and aliasing effects, thus yielding wavefront sensing exactly where diffracted light needs to be canceled. This enables picometer accuracy wavefront sensing of mid-spatial frequencies in closed loop. The wavefront reconstruction algorithm maximizes the likelihood that the acquired data corresponds to a given wavefront (the wavefront is the "free" parameter).

\subsubsection{LOW ORDER WAVEFRONT SENSING}

In order for the PIAA coronagraph to offer the smallest possible IWA, the pointing of the star on the focal plane occulter must be within a few time $10^{-3} \lambda / D$. Larger pointing errors (up to a few percent of $\lambda / D$ ) could also be allowed provided that the focal plane occulter is properly oversized, which would increase IWA.

TOPS will use stellar light reflected from the outer part of the focal plane coronagraphic mask for zerooverhead sensing. This allows sensing of low-order aberrations (such as tip-tilt and focus) before they become large enough to throw light in the science focal plane array. As shown in Figure 5, the strength of the proposed low-order wavefront sensor (LOWFS) is to produce a macroscopic intensity modulation even for very small low order aberrations. This is achieved by masking the bright central part of the PSF core (which contains a lot of light, and therefore photon noise, but little information) and only using an annulus from approximately $0.9 \lambda / \mathrm{D}$ to $1.8 \lambda / \mathrm{D}$. Two defocused images of this annulus are created on a single focal plane array to simultaneously recover approximately 10 low order modes.

Such a mask, recently integrated as part of our test-bed at the Subaru Telescope, is shown in Figure 5, along with numerical simulations of the LOWFS performance. The very center of the mask is opaque and immediately surrounding it is a reflective annulus (which appears dark gray on the image). The outer (light-colored) annulus transmits the search field from radius 1.5 to $4 \lambda / \mathrm{D}$. 

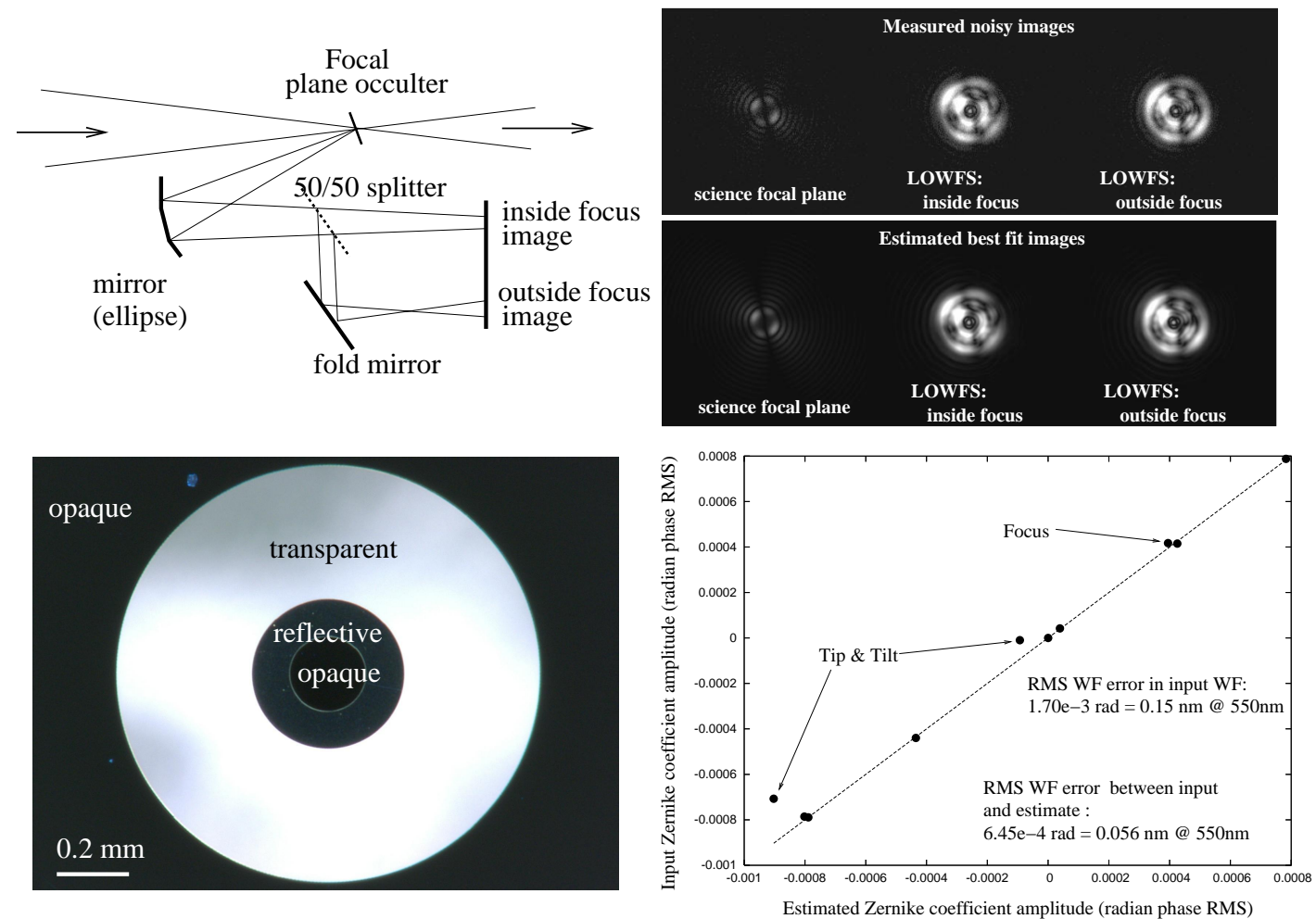

Figure 5. Noisy inside and outside focus images obtained by the LOWFS (top left). Photon noise is included in this simulation, where $10^{12}$ photons entered the telescope pupil. These images were simulated at $550 \mathrm{~nm}$ with a $50 \mathrm{pm}$ error per Zernike for the first 10 Zernikes. Wavefront reconstruction from these 2 frames accurately recovers low order wavefront aberrations (right), to an accuracy level which exceeds the requirement for $10^{10}$ contrast. Simulated science focal plane and LOWFS (bottom left), computed from the wavefront estimation, match the noisy images acquired by the LOWFS (top left).

\subsection{SPACECRAFT}

The spacecraft is designed to provide a stable environment for the TOPS optics, which is essential for successful wavefront control at the sub-nm level. Vibrations affecting mod-spatial frequencies at temporal frequencies above $\approx 0.1 \mathrm{~Hz}$ would be especially detrimental to TOPS performance, as they would be too fast to be accurately sensed and corrected by the wavefront control system (limited by photon noise).

A key design feature of the TOPS spacecraft is the Disturbance Free Payload (DFP). In this architecture, ${ }^{14}$ the Optical Telescope Assembly (OTA) and spacecraft are separate bodies: the OTA flies within the spacecraft, and the two bodies interact through non-contact sensors and actuators. The OTA is therefore isolated from spacecraft vibrations (reaction wheels) down to zero frequency, and pointing control and stability is significantly improved. High level of vibration isolation with the DFP concept has been successfully demonstrated in laboratory. ${ }^{15-17}$

\section{EXPECTED PERFORMANCE}

We show in this section that at 2-m diameter, TOPS will enable detection and characterization of Earth-like planets around about 20 main sequence stars of types F, G \& K. We discuss the assumptions and models used to derive the number shown in the above table (Sect. 3). To accurately estimate TOPS's performance, we have developed a detailed model of exo-planet imaging with a PIAA + telescope. The model produces images from which SNR can be measured, and accurately accounts for our own zodiacal light, exo-zodiacal light (assumed to be at 1 zodi level), coronagraph leakage (including stellar angular size) and orbit considerations. A detailed description of this model can be found in Guyon et. al 2006. 
We use effective exposure times which assume 100\% efficiency @ 0.55 micron in a 0.1 micron wide band. Real exposure times are probably about 10x longer due to throughput, QE and wave-front control overhead. We assume Exo-Earths to be 1 Earth Radius, with Earth albedo, at 1 AU x sqrt(star luminosity) from their star. More massive rocky planets can be detected more easily, while Mars-sized objects are significantly more challenging.

Table 2. Number of targets (stars) for which a Earth analog would be detected at SNR $=7$ as a function of telescope diameter and exposure time. The first two lines show the number of targets around which an Earth analog would be within 2 and $4 \lambda / D$. For each telescope diameter and exposure time combination, two numbers are given: the smaller number is for $50 \%$ completeness (detection probability) for a single observation; for the larger number, a $20 \%$ completeness per observation is required. Alpha Cent A and B are included in this table, but may not be suitable targets due to their duplicity and the fact that Exo-Earths might lie beyond the Outer Working Angle of the instrument. If they are to be excluded, each number in this table should be reduced by two.

\begin{tabular}{|l|c|r|r|r|r|r|}
\hline & completeness & \multicolumn{5}{|c|}{ Telescope diameter } \\
& & $1.2-\mathrm{m}$ & $1.5-\mathrm{m}$ & $2.0-\mathrm{m}$ & $3.0-\mathrm{m}$ & $4.0-\mathrm{m}$ \\
\hline \hline within $2 \lambda / \mathrm{D}$ & & 10 & 23 & 67 & 212 & 476 \\
\hline within $4 \lambda / \mathrm{D}$ & & 2 & 2 & 6 & 23 & 67 \\
\hline 1hr exposure & $50 \%$ & 2 & 3 & 7 & 24 & 51 \\
1hr exposure & $20 \%$ & 3 & 5 & 16 & 49 & 118 \\
\hline 10hr exposure & $50 \%$ & 3 & 7 & 22 & 65 & 172 \\
10hr exposure & $20 \%$ & 7 & 17 & 40 & 147 & 299 \\
\hline
\end{tabular}

There are 67 F,G,K main sequence stars for which Exo-Earths are outside $2 \lambda / \mathrm{D}$ on a 2 -m telescope at 0.55 micron. For sixteen of these 67 IWA-accessible targets, TOPS2 can expect to detect an Exo-Earth $(>20 \%$ detection probability with $1 \mathrm{hr}$ effective exposure time) and perform spectrophotometry. Seven of those are considered "easy" targets for TOPS2 (with $1 \mathrm{hr}$ effective exposure time, $>50 \%$ detection probability in a single observation). Nine out of the sixteen "realistic" targets would have their Exo-Earth separation beyond $3 \lambda / \mathrm{d}$, allowing spectrophotometry up to $\approx 0.9$ micron wavelength.

A comprehensive survey of the sixteen "realistic" TOPS2 targets would require 10 visits per target, with $10 \mathrm{hr}$ real exposure time per target. With a $100 \%$ overhead (conservative), this would require 130 days of mission time. This leaves ample time for spectrophotometry follow-up and deep observations of a larger number of targets within a realistic mission lifetime (3 to $5 \mathrm{yrs}$ ). Spectrophotometry for TOPS2 would likely be performed at the $\mathrm{R} \approx 16$ spectral resolution. This resolution is sufficient to characterize planets from their colors and detect Rayleigh scattering, but will not allow detailed chemical characterization of the planet's atmosphere/surface. Photometric monitoring of the planets may reveal a rotation period if its albedo is not geographically constant.

The numbers given in this section assume that each star has a single Earth analog, with an Earth albedo. Higher albedo planets such as Venus would be easier to detect, while Mars-like planets would be out of reach. Gas giants between 1 and $10 \mathrm{AU}$ would be particularly easy targets and could be detected around several hundred stars with a $2-\mathrm{m}$ aperture.

\section{ACKNOWLEDGMENTS}

Concept development for TOPS has been supported at Arizona by NASA under Terrestrial Planet Finder Instrument Concept Study grant NNG05GO57G. Development of the PIAA coronagraph and focal plane wavefront control techniques is supported by the National Astronomical Observatory of Japan and JPL contract numbers 1254445 and 1257767 for Development of Technologies for the Terrestrial Planet Finder Mission.

\section{REFERENCES}

[1] Kasting, J.F., Whitmire, D.P., Reynolds, R.T. 1993, Icarus, 101, 108 
[2] Kasdin, N.J., Vanderbei, R.J., Spergel, D.N., Littman, M.G. 2003, ApJ, 582, 1147

[3] Guyon, O. 2003, A\&A, 404, 379

[4] Traub, W.A., Vanderbei, R.J., 2003, ApJ, 599, 695

[5] Guyon, O., Pluzhnik, E.A., Galicher, R., Martinache, F., Ridgway, S.T., Woodruff, R.A. 2005, ApJ, 622, 744

[6] Vanderbei, R.J., Traub, W.A. 2005, ApJ, 626, 1079

[7] Martinache, F., Guyon, O., Pluzhnik, E.A., Galicher, R., Ridgway, S.T. 2006, ApJ, 639, 1129

[8] Vanderbei, R.J. 2006, ApJ, 636, 528

[9] Pluzhnik, E.A., Guyon, O., Ridgway, S.T., Martinache, F., Woodruff, R.A., Blain, C., Galicher, R. 2006, ApJ, 644, 1246

[10] Pluzhnik, E.A., Guyon, O., Warren, M., Woodruff, R.A., Ridgway, S.T. 2006, SPIE 6265-135

[11] Guyon, O., Pluzhnik, E. A., Kuchner, M. J., Collins, B., Ridgway, S. T. 2066, ApJS, 167, 81

[12] Kuchner, M.J., Crepp, J., Ge, J. 2005, ApJ, 628, 466

[13] Trauger, J.T., Traub, W.A. 2007, Nature, 446, 771

[14] Pedreiro, N. 2003, Journal of Guidance, Control and Dynamics, 26, 794

[15] Pedreiro, N., Carrier, A.C., Lorell, K.R., Roth, D.E. 2002, American Institute of Aeronautics and Astronautics, 2002-5027

[16] Gonzales, M.A., Pedreiro, N., Roth, D.E., Brookes, K., Foster, B.W. 2004, American Institute of Aeronautics and Astronautics, 2004-5247

[17] Pedreiro, N., Gonzales, M.A., Foster, B.W., Tankle, T.L., Roth, D.E. 2005, American Institute of Aeronautics and Astronautics, 2005-5876 